\title{
Probiotic effects in allergic disease
}

\begin{abstract}
The increasing prevalence of allergic disease has been linked to reduced microbial exposure in early life. Probiotics have recently been advocated for the prevention and treatment of allergic disease. This article summarises recent publications on probiotics in allergic disease, focusing on clinical studies of prevention or treatment of allergic disease. Studies employing the combined administration of pre-natal and post-natal probiotics suggest a role for certain probiotics (alone or with prebiotics) in the prevention of eczema in early childhood, with the pre-natal component of treatment appearing to be important for beneficial effects. On the other hand, current data are insufficient to support the use of probiotics for the treatment of established allergic disease, although recent studies have highlighted new hope in this area. Probiotic bacteria continue to represent the most promising intervention for primary prevention of allergic disease, and well-designed definitive intervention studies should now be a research priority.
\end{abstract}

Keyword: Allergic disease; Immune function; Intestinal microbiota; Prebiotic; Probiotic 